\title{
Genetic polymorphisms of loci D18S53, D18S59, and D18S488 in fetuses from a Chinese Tianjin Han population
}

\author{
X.Z. Li, J. Liu, Y.F. Shi, D. Ju, Y. Zhang and T.F. Yue \\ Department of Gynecology \& Obstetrics, \\ Tianjin Medical University General Hospital, Tianjin, China \\ Corresponding author: Y. Zhang \\ E-mail: yingzhangdoc@163.com
}

Genet. Mol. Res. 15 (2): gmr.15027820

Received August 8, 2015

Accepted November 26, 2015

Published June 17, 2016

DOI http://dx.doi.org/10.4238/gmr.15027820

\begin{abstract}
We investigated the genetic polymorphisms of three short tandem repeat (STR) loci, D18S53, D18S59, and D18S488, on chromosome 18 in fetuses from a Chinese Tianjin Han population. Sixty-four villus samples and 374 amniotic fluid samples were collected from fetuses. Quantitative fluorescence polymerase chain reaction was performed to amplify the STR loci, followed by scanned electrophoresis and quantitative analysis of the fluorescence signals. Hardy-Weinberg equilibrium (HWE) analysis was performed based on the genotype distributions of the STR loci to obtain the following population genetic data: genotype frequency, heterozygosity of observation $\left(H_{\mathrm{O}}\right)$, polymorphism information content (PIC), probability of discrimination power (PD), and probability of exclusion (PE). We detected 15, 13, and 15 alleles of D18S53, D18S59, and D18S488, respectively. The genotype frequencies were found to be in line with HWE. The $H_{\mathrm{O}}$ values of the three loci, D18S53, D18S59, and D18S488, were 0.797, 0.847 , and 0.792 ; the PIC values were $0.81,0.75$, and 0.73 ; the PD values were $0.944,0.901$, and 0.881 ; and the PE values were 0.593 , 0.689 , and 0.585 , respectively. D18S53, D18S59, and D18S488 loci are
\end{abstract}


good genetic markers of chromosome 18, and show potential for use in the prenatal genetic diagnosis of Edwards' syndrome.

Key words: Genetic polymorphisms; Fetus; Chromosome 18; Short tandem repeat

\section{INTRODUCTION}

Edwards' syndrome (ES) is one of the most common chromosomal abnormalities involving aneuploidy, with an incidence rate second only to that of Down's syndrome. One in 4000 individuals is born with ES, and its main manifestations include severe mental retardation and multiple malformations (Lakovschek et al., 2011; Cereda and Carey, 2012; Hurley et al., 2014). The cause of ES is still unclear and there is no effective treatment, so it is a heavy burden on the families of affected individuals, as well as on society (Sibiude et al., 2011; Wilkinson et al., 2014). Currently, the birth of infants with ES may be prevented only by effective prenatal screening and diagnosis (Elsayed et al., 2013). The rapid development of molecular biology techniques is expected to significantly improve the efficiency of ES diagnosis when applied to prenatal screening (Tekcan et al., 2014). Quantitative fluorescence polymerase chain reaction (QF-PCR) uses fluorescently labeled primers to associate a fluorescence signal with the products of amplification. The fluorescence signal, which is detected and analyzed by sensitive automated instruments, is used to quantify the peak areas of PCR amplification using genescanning software, thereby determining the original amounts of template DNA. QF-PCR is used to amplify specific short tandem repeat (STR) loci, and is therefore useful for the diagnosis of chromosomal aneuploidy and prenatal genetic diagnoses (Muthuswamy et al., 2015).

An STR is a DNA tandem repeat sequence, with two to six nucleotides forming the repeating unit. STRs contain a wealth of polymorphisms, which may indicate regional and ethnic differences (Duitama et al., 2014). STRs, also known as satellite DNA, constitute about $10 \%$ of the human genome. Satellite DNA, which is a class of highly repetitive sequences present in noncoding regions, is presumed to play a role in chromosome replication and terminal protection. An STR locus is also known as a microsatellite DNA, with a repetition number of 15 to 30 . Normally, an STR locus consists of dozens of allele fragments of lengths of about $400 \mathrm{bp}$ or less, with the most common core sequences being $(\mathrm{TG})_{\mathrm{n}},(\mathrm{AAT})_{\mathrm{n}},(\mathrm{GATA})_{\mathrm{n}}$, and $(\mathrm{CA})_{\mathrm{n}}$, which is the most common of all. These are more prevalent in untranslated regions and introns. Owing to the highly polymorphic nature and Mendelian co-dominant inheritance pattern of STR loci, these sequences are widely used in gene mapping, screening, genetic disease linkage analysis, population genetics, forensic identification of individuals, and paternity testing.

Therefore, the genetic polymorphism data provided by the typing of STR loci in different ethnic populations form the basis of chromosome aneuploidy genetic diagnosis and prenatal genetic diagnosis (Jain et al., 2010). In our previous study, we found that three STR loci on chromosome 18, namely D18S53, D18S59, and D18S488, showed genetic polymorphisms in the Tianjin Han population. They are, therefore, good candidates for use as genetic markers of chromosome 18. In this study we used QF-PCR to investigate the genetic polymorphisms of D18S53, D18S59, and D18S488 in fetuses from the Tianjin Han population, with the aim of providing experimental evidence for the prenatal diagnosis of ES. 


\section{MATERIAL AND METHODS}

\section{Subjects}

Chorionic villus samples (64) and amniotic fluid samples (374) were collected from high-risk pregnant women at 8 to 24 gestational weeks who had received treatment at the Prenatal Diagnosis Center of Tianjin Medical University General Hospital between April 2004 and October 2009. This study was conducted in accordance with the declaration of Helsinki, and received approval from the Ethics Committee of Tianjin Medical University General Hospital. All patients signed informed consent before prenatal examination.

\section{DNA extraction}

Chorionic villi $(0.5-1 \mathrm{mg})$ or amniotic fluid $(1 \mathrm{~mL})$ was taken for DNA extraction by conventional methods, as described in a previous study (Green and Sambrook, 2012).

\section{Selection of primers}

The primer sequences shown in Table 1 for the amplification of selected regions of the D18S53, D18S59, and D18S488 loci were generated using the UniSTS NCBI database (http://www.ncbi.nlm.nih.gov/unists). The 5'-end of the forward primer was labeled with the fluorescent dye caboxyfluorescein-5-succimidyl ester (FAM), and the primers were synthesized by Beijing Dingguo Biological Technology Co., Ltd. (Beijing, China).

\section{Table 1. Characteristics of the three short tandem repeat (STR) loci D18S53, D18S59, and D18S488.}

\begin{tabular}{|c|c|c|c|c|}
\hline STR locus & UniSTS No. & Chromosomal localization & Primer sequence $\left(5^{\prime}-3^{\prime}\right)$ & Fragment length (bp) \\
\hline \multirow[t]{2}{*}{ D18553 } & \multirow[t]{2}{*}{55077} & \multirow[t]{2}{*}{$18 \mathrm{p} 11.2$} & f-FAM-GGTCACCTACAACTTTGGATG & \multirow[t]{2}{*}{$159-179$} \\
\hline & & & r-TGCATGTAAATATCAGAGTCTGTT & \\
\hline \multirow[t]{2}{*}{ D18S59 } & \multirow[t]{2}{*}{14236} & \multirow[t]{2}{*}{$18 \mathrm{p} 11.32$} & f-FAM-GGTCACCTACAACTTTGGATG & \multirow[t]{2}{*}{$148-164$} \\
\hline & & & r-TGCATGTAAATATCAGAGTCTGTT & \\
\hline D18S488 & 78946 & $18 \mathrm{q} 22.3$ & $\begin{array}{l}\text { f-FAM-TTCTGAAGACAGATCCAAGTG } \\
\text { r-ATCATGTGAGCCAATTCCT }\end{array}$ & $239-264$ \\
\hline
\end{tabular}

\section{PCR amplification}

The total volume of the PCR mixture (Takara, Tokyo, Japan) was $25 \mu \mathrm{L}$, including $1 \mathrm{X}$ PCR buffer, $0.2 \mathrm{mM}$ dNTPs, $0.04 \mathrm{U} / \mu \mathrm{L}$ Taq enzyme, and $\sim 0.6 \mathrm{ng}$ DNA template, with a primer concentration of $0.12 \mu \mathrm{M}$. The PCR amplification conditions were: pre-denaturation at $94^{\circ} \mathrm{C}$ for $2 \mathrm{~min}$; 35 cycles of denaturation at $94^{\circ} \mathrm{C}$ for $30 \mathrm{~s}$, annealing at $52^{\circ} \mathrm{C}$ for $30 \mathrm{~s}$, and extension at $72^{\circ} \mathrm{C}$ for $1 \mathrm{~min}$; followed by extension at $72^{\circ} \mathrm{C}$ for $10 \mathrm{~min}$ and storage at $4^{\circ} \mathrm{C}$.

The amplification products were detected by $4 \%$ polyacrylamide gel electrophoresis. An ABI PRISM 377 automatic sequencer was used to scan the electropherogram, with the GeneScan 3.1 software used for data extraction. The Matrix file of plastic films was installed, the appropriate internal standard was selected, and analysis parameters were set. The BinThere software was used to set the fragment sizes, and the resulting data were imported into the GeneScan 3.1 software, so that the corresponding peak spectra and related data could be 
obtained. The sizes and peak areas of the fluorescence signals from the DNA fragments from electrophoresis were analyzed, according to the positions of intra-molecular electrophoresis markers. The alleles were then numbered in ascending order so that the genotype of each sample could be obtained.

\section{Statistical analysis}

The direct counting method was used to determine the allele frequency for each locus, which was then analyzed by the SPSS13.0 software to verify whether it was in agreement with the Hardy-Weinberg equilibrium (HWE) $(\mathrm{P}>0.05)$. The mean, standard deviation, and $95 \%$ confidence intervals $(95 \% \mathrm{CIs})$ for the reference range of peak area ratios were statistically analyzed for the heterozygous individual. The PowerStatsV12 software was used for statistical analysis, and the heterozygosity of observation $\left(H_{\mathrm{O}}\right)$, polymorphism information content (PIC), probability of discrimination power (PD), and probability of exclusion (PE) were calculated for the three STR loci.

\section{RESULTS}

\section{Allele frequencies of the three loci}

All 438 samples were successfully amplified. Fifteen alleles were found at the D18S53 STR locus, with allele fragment lengths ranging from 161 to $185 \mathrm{bp}$. These fragments were named 1 to 15 , from the shortest to the longest. A total of 52 genotypes were found, the frequencies of which were consistent with $\operatorname{HWE}\left(\chi^{2}=23.94, \mathrm{P}=1.00\right)$. Thirteen alleles were found at the D18S59 STR locus, with allele fragment lengths ranging from 128 to $152 \mathrm{bp}$. These were named 1 to 13 , from the shortest to the longest. A total of 47 genotypes were found, whose frequencies were consistent with $\operatorname{HWE}\left(\chi^{2}=52.10, \mathrm{P}=0.19\right)$. Fifteen alleles were found at the D18S488 STR locus, with allele fragment lengths ranging from 236 to 264 $\mathrm{bp}$. These were named 1 to 15 , from the shortest to the longest. A total of 42 genotypes were found, whose distribution was in agreement with $\operatorname{HWE}\left(\chi^{2}=45.11, \mathrm{P}=0.23\right)$. The allele frequencies for the three STR loci are shown in Table 2. The electrophoresis and GeneScan analysis results for each allele are shown in Figures 1-4.

Table 2. Allele frequencies of the D18S53, D18S59, and D18S488 loci.

\begin{tabular}{l|c|c|c}
\hline Allele fragment & Allele frequency of D18S53 & Allele frequency of D18S59 & Allele frequency of D18S488 \\
\hline 1 & 0.0011 & 0.0034 & 0.0067 \\
\hline 2 & 0.0067 & 0.0157 & 0.2864 \\
\hline 3 & 0.0011 & 0.0145 & 0.0895 \\
\hline 4 & 0.0302 & 0.0168 & 0.0045 \\
\hline 5 & 0.0190 & 0.3982 & 0.0213 \\
\hline 6 & 0.2092 & 0.0570 & 0.0034 \\
\hline 7 & 0.1544 & 0.1208 & 0.0011 \\
\hline 8 & 0.2472 & 0.1141 & 0.0067 \\
\hline 9 & 0.1633 & 0.0190 & 0.0011 \\
\hline 10 & 0.0772 & 0.1767 & 0.3859 \\
\hline 11 & 0.0459 & 0.0336 & 0.1723 \\
\hline 13 & 0.0112 & 0.0246 & 0.0291 \\
\hline 14 & 0.0101 & 0.0067 & 0.0179 \\
\hline 15 & 0.0011 & & 0.0034 \\
\hline
\end{tabular}




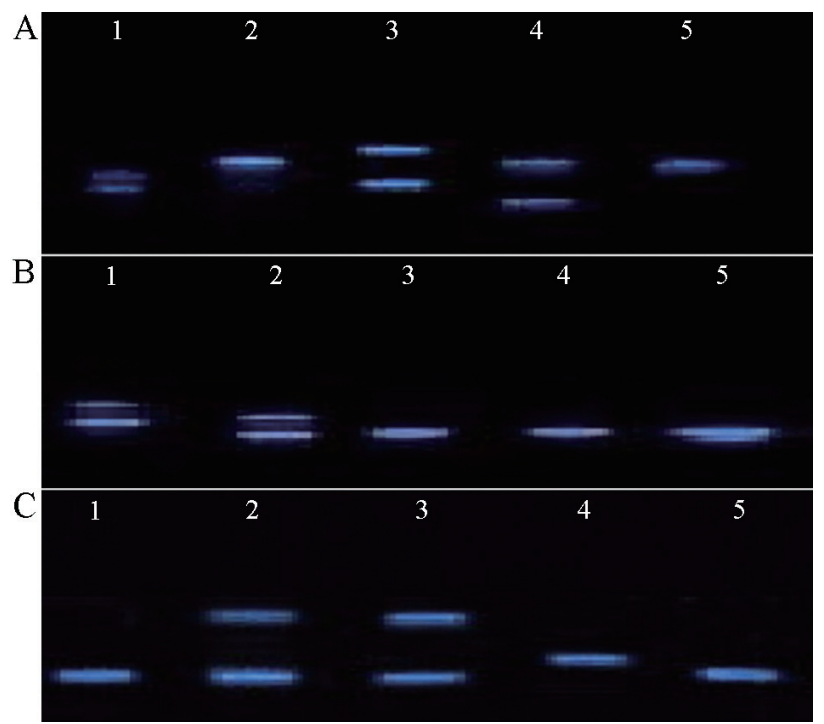

Figure 1. Electrophoresis result for the D18S53 (A), D18S59 (B), and D18S488 (C) loci. Locus D18S53 (A) exhibited two fluorescent bands in lanes 1, 3 and 4, while it is shown signal fluorescent band in lanes 2 and 5. Locus D18S59 (B) exhibited two fluorescent bands in lanes 1 and 2, while it is shown signal fluorescent band in lanes 3, 4 and 5. Locus D18S488 (C) exhibited two fluorescent bands in lanes 2 and 3, while it is shown signal fluorescent band in lanes 1,4 and 5 .

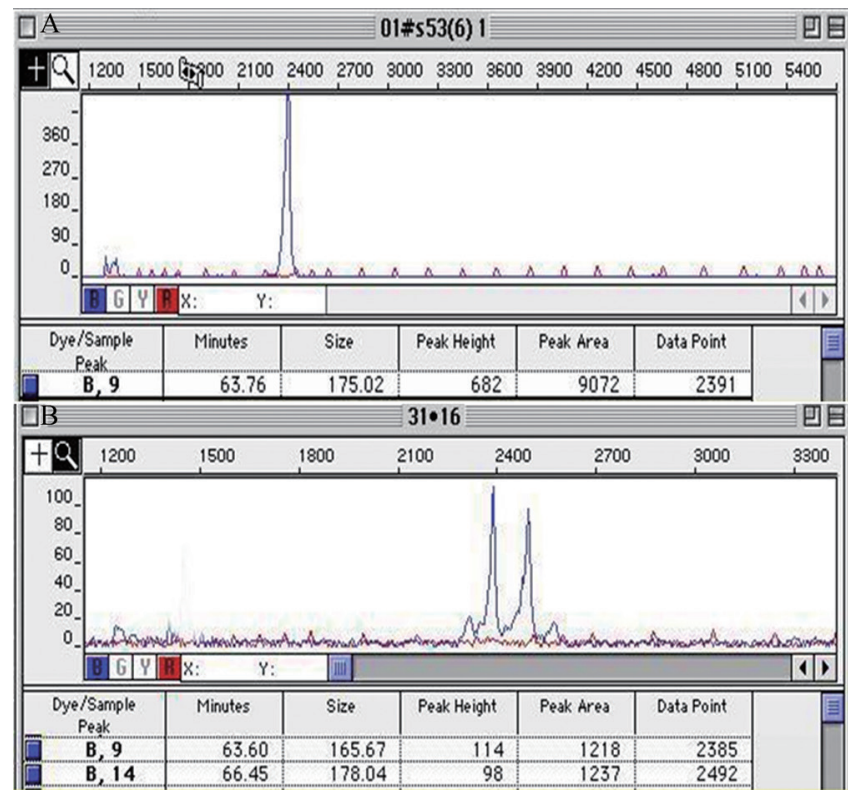

Figure 2. GeneScan result for the D18S53 locus. A. and B. are the GeneScan analysis results for lanes 2 and 3 of the D18S53 locus, respectively; lane 2 exhibited the corresponding fluorescent signal peaks; lane 3 exhibited 2 peaks, with a peak area ratio of approximately 1:1 (1218:1237). 


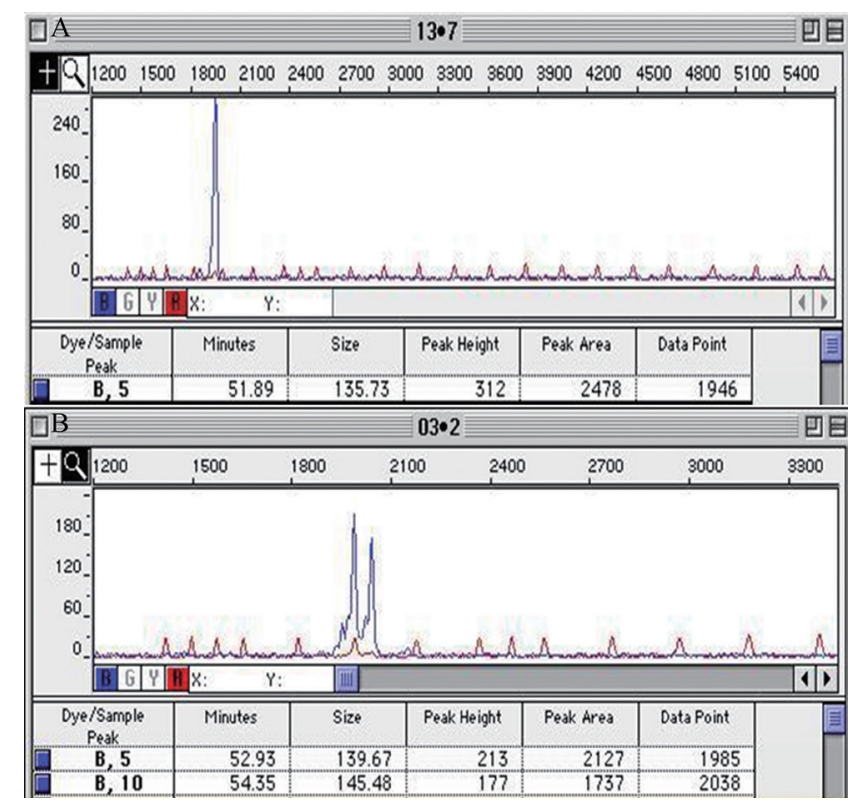

Figure 3. GeneScan result for the D18S59 locus. A. and B. are the GeneScan analysis results for lanes 2 and 3 of the D18S59 locus, respectively; lane 2 exhibited the corresponding fluorescent signal peaks; lane 3 exhibited 2 peaks, with a peak area ratio of approximately1:1 (2127:1737).

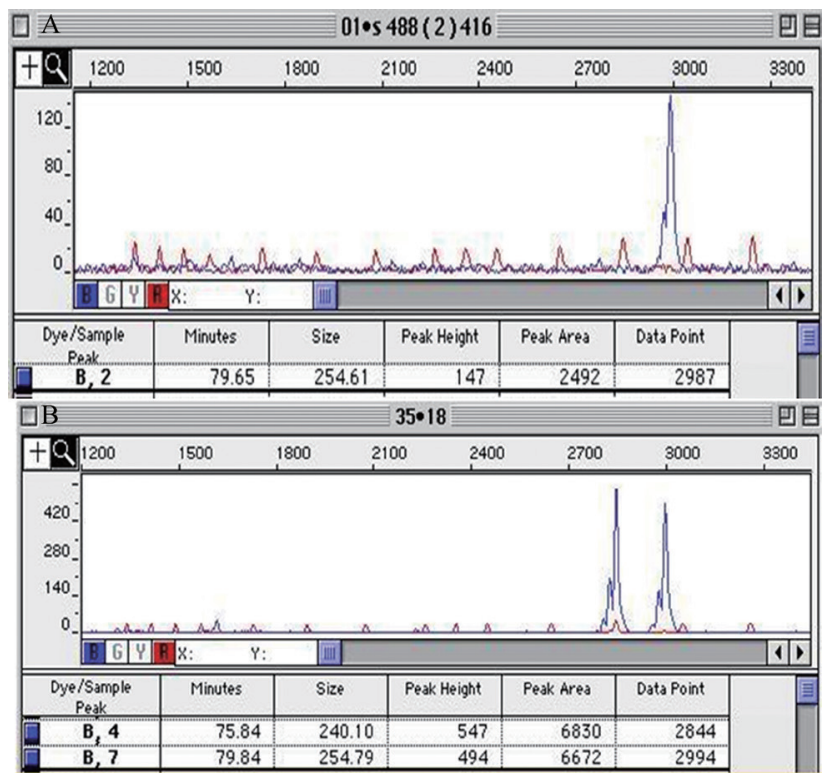

Figure 4. GeneScan result for the D18S488 locus. A. and B. are the GeneScan analysis results for lanes 4 and 3 of the D18S488 locus, respectively; lane 4 exhibited the corresponding fluorescent signal peaks; lane 3 exhibited 2 peaks, with a peak area ratio of approximately 1:1 (6830:6672). 


\section{Genetic polymorphism}

Values for the statistical genetic polymorphism parameters $\left(H_{\mathrm{O}}, \mathrm{PIC}, \mathrm{DP}\right.$, and PE) of the three STR loci, D18S53, D18S59, and D18S488, in fetuses of Tianjin Han ethnic background are shown in Table 3.

Table 3. Genetic polymorphism parameters of the three short tandem repeat (STR) loci.

\begin{tabular}{l|c|c|c|c}
\hline Locus & $H_{\mathrm{O}}$ & PIC & PD & PE \\
\hline D18S53 & 0.797 & 0.81 & 0.944 & 0.593 \\
\hline D18S59 & 0.847 & 0.75 & 0.901 & 0.689 \\
\hline D18S488 & 0.792 & 0.73 & 0.881 & 0.585 \\
\hline
\end{tabular}

$H_{\mathrm{O}}=$ heterozygosity of observation; $\mathrm{PIC}=$ polymorphism information content; $\mathrm{PD}=$ probability of discrimination power; $\mathrm{PE}=$ probability of exclusion.

\section{DISCUSSION}

In QF-PCR, the template DNA is amplified by PCR using fluorescently labeled primers, so that the fluorescent label is incorporated into the PCR amplification products. Therefore, the fluorescent signal generated is proportional to the amount of amplification product. After the collection and analysis of fluorescence data by automated equipment of sufficient sensitivity, the peak areas of corresponding repeated length within DNA sequences are determined by the gene scanning software, thereby quantifying the original template DNA (Okamoto et al., 2003). In 1991, Edwards et al. first applied STR-PCR to establish second-generation DNA fingerprinting technology for the diagnosis of X chromosome monomer disease. Mansfield (1993) subsequently used STR loci as genetic markers for chromosome 21, and performed QF-PCR for the genetic diagnosis of patients with Down's syndrome. Several studies have since utilized the unique advantages of STR loci for the diagnosis of abnormal chromosome numbers (Langlois and Duncan, 2011; Papageorgiou et al., 2011).

QF-PCR has been widely used for the rapid diagnosis of common chromosome aneuploidies in the USA, the UK, and in other European countries (Mann and Ogilvie, 2012). Hills et al. (2010) retrospectively analyzed 737 prenatal diagnostic specimens in region 9 of London, and found that 73 cases with serious maternal contamination could be analyzed by QF-PCR alone. Compared with conventional cytogenetic prenatal diagnosis, QF-PCR has the advantages of being faster, more convenient, and cost-effective when applied to prenatal diagnosis (Jenderny et al., 2014). In this study, three STR loci were selected from near the centromere, from the short arm, and from the long arm of chromosome 18, with the aim of providing experimental evidence for ES gene diagnosis and prenatal diagnosis in fetuses of Tianjin Han origin. Through the statistical analysis of the QF-PCR results, this study provides the experimental basis for establishing the diagnostic criteria of this method, with applications in genetic and prenatal diagnoses.

Currently, there are few reports about the population genetic data for D18S53, D18S59, and D18S488, either in China or globally. In this study we found that in fetuses of Tianjin Han nationality, the three STR loci, D18S53, D18S59, and D18S488, had $H_{\mathrm{O}}$ values of $0.797,0.847$, and 0.792 ; PIC values of $0.81,0.75$, and 0.73 ; PD values of $0.944,0.901$, and 0.881 ; and PE values of $0.593,0.689$, and 0.585 , respectively. These results are consistent with the population genetic data for D18S53, D18S59, and D18S488 in the Tianjin Han population 
from our previous studies. For the STR loci selected in this study, $H_{\mathrm{O}}>0.7$, PIC $>0.5$, PD $>0.8$, and $\mathrm{PE}>0.5$, indicating that D18S53, D18S59, and D18S488 may be used as reliable genetic markers of chromosome 18, as well as for prenatal genetic diagnosis of ES.

The QF-PCR technique has received much attention owing to its cost-effectiveness and efficiency. Gekas et al. (2011) studied the prenatal diagnostic programs for 110,948 pregnant women and found that QF-PCR could save US\$3494 in diagnosing a single Down's syndrome case, compared with karyotype analysis. Therefore, it is necessary to establish a QF-PCR diagnostic program in line with the population characteristics of Tianjin. As the same STR locus may contain alleles with different lengths in different individuals, QF-PCR amplification may indicate two different situations based on STR amplification: the homozygotes show one band/peak and the heterozygotes show two bands/peaks, with a quantitative ratio of about 1:1. In this study, three STR loci (in a total of 1067 samples) indicated heterozygotes, with the peak area ratio of those two peaks of $0.50-1.76$, mean $\pm \mathrm{SD}=1.06 \pm 0.21$, and $95 \% \mathrm{CI}=$ 1.06-0.41. The quantitative results of QF-PCR peak area analysis showed the peak area ratio to be $0.55-1.47$, within the normal reference range and consistent with the results obtained by Putzova et al. (2008).

This study found that loci D18S53, D18S59, and D18S488 on chromosome 18 had higher heterozygosity in fetuses of Tianjin Han background, suggesting that these may be used as good genetic markers of chromosome 18. The quantitative results for the QF-PCR peak area were within the normal reference range, thus providing a theoretical basis for the further genetic diagnosis and prenatal genetic diagnosis of ES. Follow-up research should aim to continue to screen STR loci on chromosomes 13, X, and Y for the diagnosis of abnormalities in these chromosomes.

\section{Conflicts of interest}

The authors declare no conflict of interest.

\section{ACKNOWLEDGMENTS}

Research supported by the Tianjin Scientific and Technological Project (\#06YFSYSF01800), and the Tianjin Education Commission Foundation Project (\#20050229).

\section{REFERENCES}

Cereda A and Carey JC (2012). The trisomy 18 syndrome. Orphanet J. Rare Dis. 7: 81. http://dx.doi.org/10.1186/1750$\underline{1172-7-81}$

Duitama J, Zablotskaya A, Gemayel R, Jansen A, et al. (2014). Large-scale analysis of tandem repeat variability in the human genome. Nucleic Acids Res. 42: 5728-5741. http://dx.doi.org/10.1093/nar/gku212

Edwards A, Civitello A, Hammond HA and Caskey CT (1991). DNA typing and genetic mapping with trimeric and tetrameric tandem repeats. Am. J. Hum. Genet. 49: 746-756.

Elsayed GM, El Assiouty L and El Sobky ES (2013). The importance of rapid aneuploidy screening and prenatal diagnosis in the detection of numerical chromosomal abnormalities. Springerplus 2: 490. http://dx.doi.org/10.1186/2193-1801$\underline{2-490}$

Gekas J, van den Berg DG, Durand A, Vallée M, et al. (2011). Rapid testing versus karyotyping in Down's syndrome screening: cost-effectiveness and detection of clinically significant chromosome abnormalities. Eur. J. Hum. Genet. 19: 3-9. http://dx.doi.org/10.1038/ejhg.2010.138 
Green M and Sambrook J (2012). Molecular cloning: a laboratory manual. 4th edn. Cold Spring Harbor Laboratory Press, Cold Spring Harbor, NY.

Hills A, Donaghue C, Waters J, Waters K, et al. (2010). QF-PCR as a stand-alone test for prenatal samples: the first 2 years' experience in the London region. Prenat. Diagn. 30: 509-517.

Hurley EH, Krishnan S, Parton LA and Dozor AJ (2014). Differences in perspective on prognosis and treatment of children with trisomy 18. Am. J. Med. Genet. A. 164A: 2551-2556. http://dx.doi.org/10.1002/ajmg.a.36687

Jain S, Agarwal S, Panigrahi I, Tamhankar P, et al. (2010). Diagnosis of Down syndrome and detection of origin of nondisjunction by short tandem repeat analysis. Genet. Test. Mol. Biomarkers 14: 489-491. http://dx.doi.org/10.1089/ gtmb.2009.0191

Jenderny J, Schmidt W and Kochhan L (2014). Chromosome aberrations identified by cytogenetic analysis of the first two clones of cultured amniotic fluid cells compared with QF-PCR results. Cytogenet. Genome Res. 142: 239-244. http:// dx.doi.org/10.1159/000362524

Lakovschek IC, Streubel B and Ulm B (2011). Natural outcome of trisomy 13, trisomy 18, and triploidy after prenatal diagnosis. Am. J. Med. Genet. A. 155A: 2626-2633. http://dx.doi.org/10.1002/ajmg.a.34284

Langlois S and Duncan A (2011). Use of a DNA method, QF-PCR, in the prenatal diagnosis of fetal aneuploidies. $J$. Obstet. Gynaecol. Can. 33: 955-960. http://dx.doi.org/10.1016/S1701-2163(16)35022-8

Mann K and Ogilvie CM (2012). QF-PCR: application, overview and review of the literature. Prenat. Diagn. 32: 309-314. http://dx.doi.org/10.1002/pd.2945

Mansfield ES (1993). Diagnosis of Down syndrome and other aneuploidies using quantitative polymerase chain reaction and small tandem repeat polymorphisms. Hum. Mol. Genet. 2: 43-50. http://dx.doi.org/10.1093/hmg/2.1.43

Muthuswamy S, Bhalla P, Agarwal S and Phadke SR (2015). Performance of QF-PCR in targeted prenatal aneuploidy diagnosis: Indian scenario. Gene 562: 55-61. http://dx.doi.org/10.1016/j.gene.2015.02.028

Okamoto O, Yamamoto Y, Inagaki S, Yoshitome K, et al. (2003). Analysis of short tandem repeat (STR) polymorphisms by the powerplex 16 system and capillary electrophoresis: application to forensic practice. Acta Med. Okayama 57: 59-71.

Papageorgiou EA, Karagrigoriou A, Tsaliki E, Velissariou V, et al. (2011). Fetal-specific DNA methylation ratio permits noninvasive prenatal diagnosis of trisomy 21. Nat. Med. 17: 510-513. http://dx.doi.org/10.1038/nm.2312

Putzova M, Soldatova I, Pecnova L, Dvorakova L, et al. (2008). QF-PCR-based prenatal detection of common aneuploidies in the Czech population: five years of experience. Eur. J. Med. Genet. 51: 209-218. http://dx.doi.org/10.1016/j. ejmg.2008.02.003

Sibiude J, Gavard L, Floch-Tudal C and Mandelbrot L (2011). Perinatal care and outcome of fetuses with trisomies 13 and 18 following a parental decision not to terminate the pregnancy. Fetal Diagn. Ther. 29: 233-237. http://dx.doi. org $/ 10.1159 / 000322133$

Tekcan A, Tural S, Elbistan M, Kara N, et al. (2014). The combined QF-PCR and cytogenetic approach in prenatal diagnosis. Mol. Biol. Rep. 41: 7431-7436. http://dx.doi.org/10.1007/s11033-014-3630-7

Wilkinson DJ, de Crespigny L, Lees C, Savulescu J, et al. (2014). Perinatal management of trisomy 18: a survey of obstetricians in Australia, New Zealand and the UK. Prenat. Diagn. 34: 42-49. http://dx.doi.org/10.1002/pd.4249 